\author{
退火对单层二硫化钼荧光特性的影响 \\ 沈成 ${ }^{a}$ 张菁 $a$ 时东霞*, $a$ 张广宇*, $a, b$ \\ $\left({ }^{a}\right.$ 中国科学院物理研究所 北京凝聚态物理国家实验室 北京 100190) \\ $\left({ }^{b}\right.$ 量子物质科学协同创新中心 北京 100190$)$
}

\begin{abstract}
摘要 单层二硫化钿是继石墨烯后的一种新型二维材料. 它是一种直接带隙半导体, 具有优异的光电特性, 从而受到 人们的广泛关注. 之前的研究报道过单层二硫化钿在氞气中退火后可以提升其 $\mathrm{A}$ 激子峰的苂光强度, 但我们发现, 空 气中退火较氩气退火效果更为明显. 本文重点研究了在空气中退火对二硫化钼的荧光特性的影响. 不同条件下制备的 单层二硫化锄样品, 经过在空气中退火处理后, 荧光峰位均发生了蓝移, 荧光强度提升了一个数量级. 我们认为, 这是 由于空气退火造成二硫化钿缺陷的形成, 大量氧气分子被缺陷束缚并发生电荷转移. 氧气分子充当受主的角色, 起着 $\mathrm{P}$ 型掺杂的作用. 电荷的抽取造成二硫化钿的负电激子减少，中性激子增多，提升了其苂光量子效率. 我们在对照实验 中发现, $\mathrm{NH}_{3}$ 吸附在二硫化钿表面时, 荧光强度下降, 峰位红移, 这是由于 $\mathrm{NH}_{3}$ 分子充当施主的角色, 造成负电激子增 多, 中性激子减少. 本文为提高单层二硫化锄的荧光量子效率提供了一种简单有效的方法.
\end{abstract}

关键词 二硫化钼; 苂光; 激子; 电荷转移

\title{
Photoluminescence Enhancement in Monolayer Molybdenum Disulfide by Annealing in Air
}

\author{
Shen, Cheng ${ }^{a} \quad$ Zhang, Jing $^{a} \quad$ Shi, Dongxia* ${ }^{* a} \quad$ Zhang, Guangyu ${ }^{*, a, b}$ \\ ( ${ }^{a}$ Beijing National Laboratory for Condensed Matter Physics and Institute of Physics, \\ Chinese Academy of Sciences, Beijing 100190) \\ $\left({ }^{b}\right.$ Collaborative Innovation Center of Quantum Matter, Beijing 100190)
}

\begin{abstract}
Monolayer molybdenum disulfide is a novel two-dimensional material beyond graphene. It is a direct band gap semiconductor with excellent electrical and optical properties, promising wide application in nanoelectronics and optoelectronics, thus has drawn much attention recently. In this paper, we investigate the enhancement of photoluminescence of monolayer molybdenum disulfide by annealing in air. Monolayer molybdenum disulfide samples were prepared by mechanical exfoliation and chemical vapor deposition with molybdenum oxide and sulfur as sources, and argon as carrier gas. We found that air annealing for several minutes can distinctly enhance the photoluminescence intensity of A exciton by an order of magnitude, which is much better than annealing in argon. The blue shift of A exciton peak is observed after air annealing for all the monolayer molybdenum disulfide samples prepared by different methods above. We also found that this phenomenon widely exists in samples with different substrates like silicon dioxide and sapphire. Electrical transport measurements were carried out and indicate that the carrier mobility of monolayer molybdenum disulfide is largely reduced after annealing in air, which might mean the formation of considerable defects. This phenomenon is believed to be due to the doping effect caused by adsorption of oxygen, which is bonded to the defects after annealing. As acceptors, these oxygen dopants change the distribution of trions and neutral excitons, resulting in less tritons but more neutral excitons. Neutral excitons yield much higher quantum efficiency. As a comparison, we carried out control experiments in which annealing was in $\mathrm{NH}_{3}$ atmosphere. We found that photoluminescence is red shifted and can be quenched. This research results give a simple and effective method to enhance the photoluminescence of monolayer molybdenum disulfide.

Keywords molybdenum disulfide; photoluminescence; exciton; charge transfer
\end{abstract}

\section{1 引言}

近几年来, 二维材料由于其可调的光学和电学特性 受到了人们的广泛关注和研究兴趣 ${ }^{[1 \sim 4]}$. 相比石墨烯的 金属性，过渡金属硫化物(TMDCs)，包括 $\mathrm{MoS}_{2}, \mathrm{WS}_{2}$,
$\mathrm{MoSe}_{2}, \mathrm{WSe}_{2}$, 具有能隙, 弥补了石墨烯在光电方面应 用的受限, 表现出优异的光学和电学性能, 成为近期的 研究热点 ${ }^{[1]}$. 多层二硫化钿为间接带隙半导体, 当层数 减至一层时转变为直接带隙半导体 ${ }^{[5,6]}$, 从而极大地提 高了 $\mathrm{MoS}_{2}$ 的光电响应，拓宽了其在光电探测、太阳能

*E-mail: gyzhang@iphy.ac.cn; dxshi@iphy.ac.cn

Received March 31, 2015; published June 1, 2015.

Project supported by the National Basic Research Program of China (No. 2013CB934500) and the National Natural Science Foundation of China (Nos. $61390503,61325021,91223204,91323304)$.

项目受科技部 973 项目(No. 2013CB934500)和国家自然科学基金(Nos. 61390503, 61325021, 91223204, 91323304)资助. 
电池、气体探测、生物传感等方面的应用.

有研究报道，二硫化钼表面残留的 $\mathrm{Li}^{+}$可以填充间 隙产生应力从而导致二硫化钼拉曼光谱中 $\mathrm{E}_{2 \mathrm{~g}}^{1}$ 和 $\mathrm{A}_{1 \mathrm{~g}}$ 峰 位的改变和强度的降低 ${ }^{[7]}$; 基于二硫化钼的晶体管在水 分存在的环境下, 表现出电流-电压回滞现象 ${ }^{[8]}$; NO 的 P 型掺杂, 可以改变二硫化钼 $\mathrm{N}$ 型半导体的阻值 ${ }^{[9]}$.

由于直接带隙的形成, 相比多层 $\mathrm{MoS}_{2}$, 单层 $\mathrm{MoS}_{2}$ 的苂光强度得到了极大的增强, 但是其苂光量子产率仍 有待提高 ${ }^{[6]}$. 二硫化钼的荧光响应来自于体内的 A、B 激子的产生和复合 ${ }^{[6]}$ (自旋-轨道的耦合作用使价带顶䢃 裂, 造成了两种激子的形成). 而最近的研究表明, 电子 可以被激子束缚形成负电激子, 从而改变二硫化钼苂光 光谱的特点 ${ }^{[10]}$. 二硫化钼的电子分布可以通过栅极电 压、化学掺杂、分子吸附等手段进行调控 ${ }^{[11 ~ 13]}$. 有文献 报道, 气体分子的电荷转移作用可以极大地改变 TMDCs 的苂光光谱 ${ }^{[14,15]}$. 气体分子吸附导致荧光光谱 的强烈变化为 TMDCs 在气体探测甚至是生物传感上的 应用打开了一扇大门. 在本文中, 我们提出了空气退火 可以显著地提高二硫化钼的荧光量子效率, 增强其荧光 强度的观点，并对其机制作出了阐述.

\section{2 结果与讨论}

单层 $\mathrm{MoS}_{2}$ 样品是采用化学气相沉积方法(CVD)在 $300 \mathrm{~nm} \mathrm{\textrm {SiO } _ { 2 }}$ 衬底上生长的, 具体生长方法可以参照我 们前期关于三温区 CVD 生长二硫化钼的介绍 ${ }^{[16]}$. 在管 式炉中空气退火的条件是: 升温至 $250{ }^{\circ} \mathrm{C}$ 保持 $12 \mathrm{~min}$. 图 1(a)和 1(b)分别对应样品退火前后的 AFM 形貌像. 生 长的 $\mathrm{MoS}_{2}$ 为单层连续膜, 晶界不明显, 有一些零散分 布的第二层的核, 样品在空气中退火处理后表面形貌没 有发生明显变化. 图 1(c)是样品退火前后的拉曼谱, 基 本没有发生变化. 而从样品退火前后的荧光谱比较发现 [图 1(d)], 当样品退火后, A 激子峰荧光强度急剧上升 (大约上升一个量级), 且半峰宽明显下降, 峰形蓝移, 而 $\mathrm{B}$ 激子峰强度无较大变化. 有其他文献称这种现象是 $\mathrm{B}$ 激子峰消失在 $\mathrm{A}$ 激子峰中 ${ }^{[17]}$. 样品处理前后的拉曼光 谱和 AFM 表征显示, 样品在处理后表面形貌和晶格振 动基本没有发生明显变化, 无晶界开裂的现象. 因此, 空气中退火后苂光谱的蓝移和增强可以初步排除应力 导致晶界开裂的因素.

通常, 物质的荧光强度主要取决于三个方面: 电子 密度、电子跃迁几率、无辐射弛豫的相对比例. 其中电 子跃迁几率主要与带隙种类(间接带隙或直接带隙)有 关, 电子密度与材料的本征性质、掺杂浓度有关, 无辐 射弛豫的相对比例与引入的杂质能级、材料的质量等有 关. 真空退火或者氩气退火可以起到清洁表面、减少晶 格缺陷的作用, 从而在一定程度上提高苂光强度. 为了 验证苂光增强是否与空气中退火的清洁作用和缺陷减
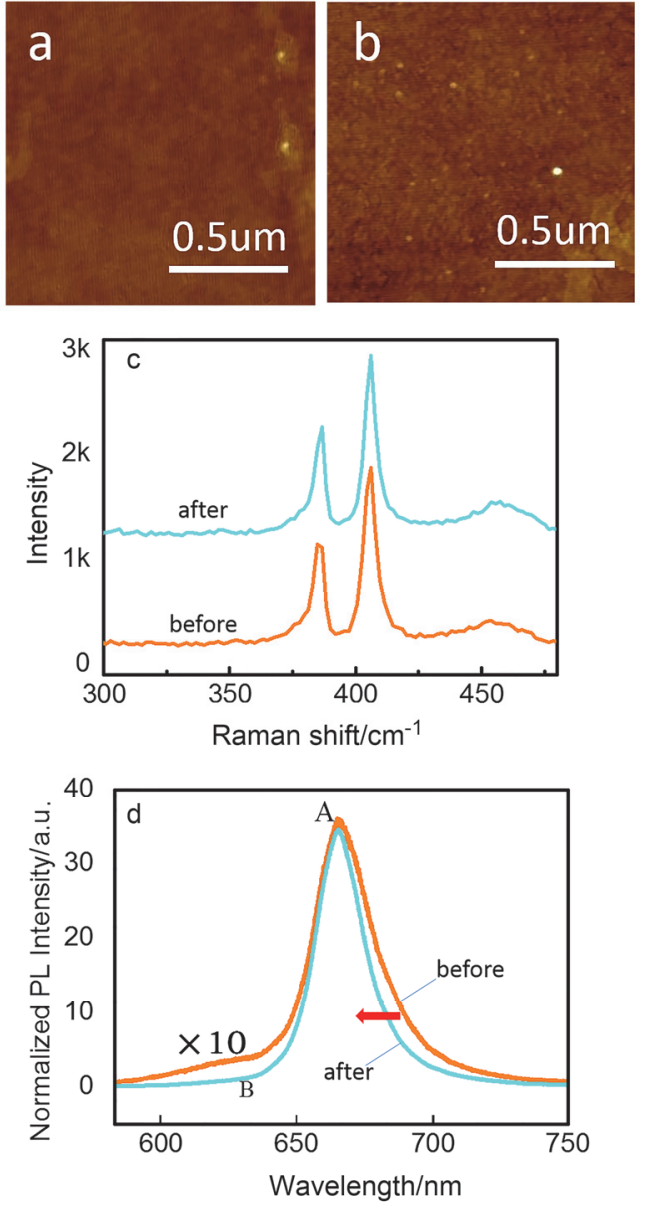

图 1 (a), (b)分别为 $\mathrm{CVD}$ 生长在 $\mathrm{SiO}_{2}$ 祄底上的单层二硫化锄样品退 火前后的 AFM 图像, 退火后并未观察到晶界开裂的现象; (c)该样品 退火前后的拉曼光谱, 无明显差异; (d)样品退火前后的荧光光谱, 橙 色、蓝色曲线分别代表退火前、退火后的荧光光谱; 图中退火前的苂 光曲线放大了 10 倍; 退火后右半峰明显变窄，峰形整体向高能区收 缩, 如图中的红色箭头所示

Figure 1 (a) AFM image of CVD grown monolayer $\mathrm{MoS}_{2}$ on $\mathrm{SiO}_{2}$ before annealing. (b) AFM image of CVD grown monolayer $\mathrm{MoS}_{2}$ on $\mathrm{SiO}_{2}$ after annealing, with no sign of grain boundary separation. (c) Raman spectrum of $\mathrm{MoS}_{2}$ before and after annealing with no difference. (d) PL spectrum of annealed and non-annealed $\mathrm{MoS}_{2}$, represented by blue and orange curve respectively. The orange line is magnified by 10 times. It's obvious that the peak contracts to higher energy as the red arrow shows

少有关, 我们和在氩气中退火的样品做了比较. 在氩气 经过同样退火处理 $\left(250{ }^{\circ} \mathrm{C}, 10 \mathrm{~min}\right)$ 的样品, 其苂光强度 并未增强. 延长在氩气中的退火时间(第一次为 $250{ }^{\circ} \mathrm{C}$, $30 \mathrm{~min}$; 第二次为 $300{ }^{\circ} \mathrm{C}, 10 \mathrm{~h}$ )时, 其苂光强度有较大 增强, 但和空气退火处理 $\left(250{ }^{\circ} \mathrm{C}, 8 \mathrm{~min}\right)$ 的样品比较, 这种荧光增强的效率明显低很多, 见图 2(a). 因此空气 退火对单层 $\mathrm{MoS}_{2}$ 苂光的明显增强可以排除表面清洁的 作用机制.

另外，苂光的增强是否与祄底或是样品的制备方法 有关，我们也研究了 CVD 方法生长在蓝宝石祄底上的 单层 $\mathrm{MoS}_{2}$ 样品[图 2(b)] 和机械剥离在 $300 \mathrm{~nm} \mathrm{SiO} 2$ 上的 

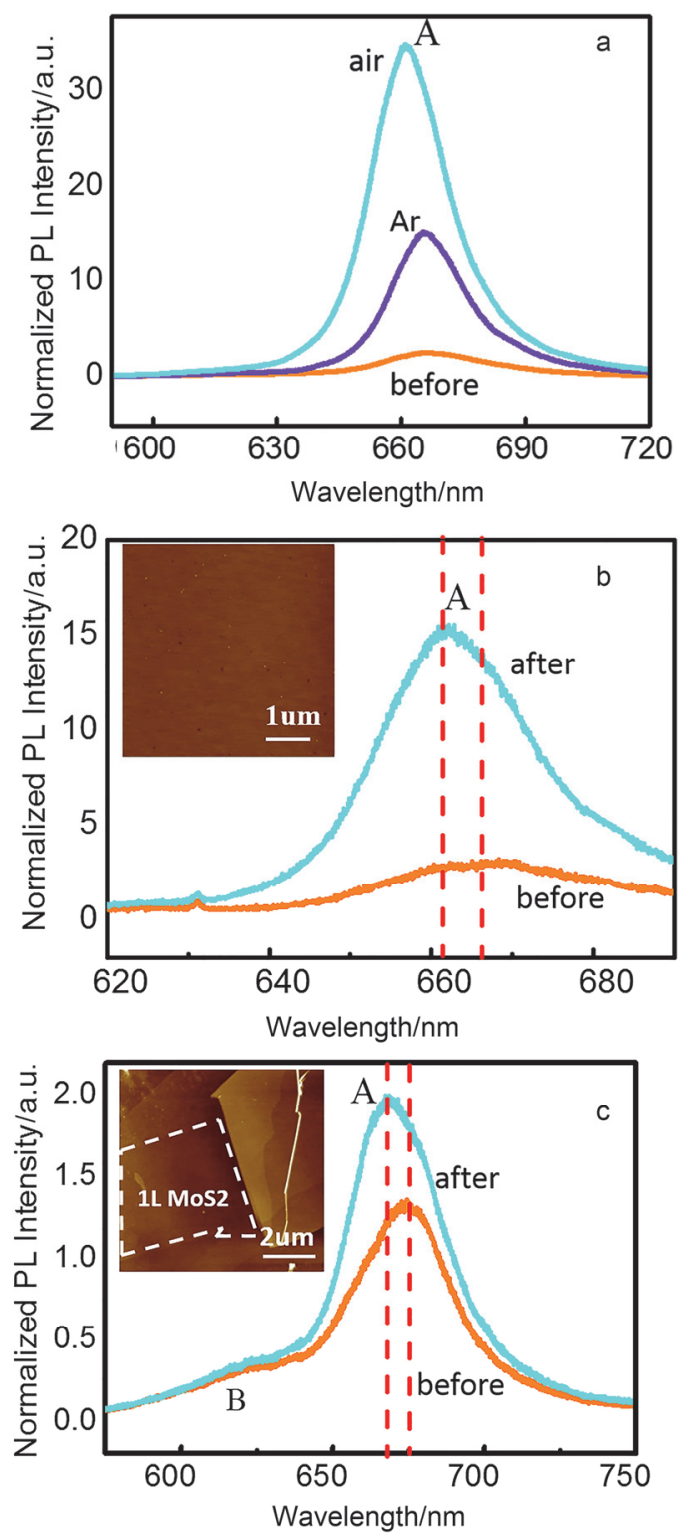

图 2 (a)氩气和空气退火的比较, 橙色、紫色、蓝色曲线分别代表退 火前、氩气 $300{ }^{\circ} \mathrm{C}$ 退火 $10 \mathrm{~h}$ 、空气 $250{ }^{\circ} \mathrm{C}$ 退火 $8 \mathrm{~min}$ 后的荧光光谱; (b) CVD 方法生长在蓝宝石祄底上的单层二硫化钼在空气中退火前(橙 色)和退火后(蓝色)的苂光光谱, 插图为样品的 AFM 扫描图; (c)机械 剥离在 $\mathrm{SiO}_{2}$ 的单层二硫化钼在空气中退火前(登色)和退火后(蓝色)的 苂光光谱，插图白色虚线区域为单层二硫化钼

Figure 2 (a) The comparison of annealing in Ar and air. The contrast of PL intensity between non-annealed, annealed in Ar for 10 hours and annealed in air for 8 minutes samples is shown by orange, purple and blue curve, respectively. (b) PL spectrum of CVD grown monolayer $\mathrm{MoS}_{2}$ on sapphire. Orange and blue represent before and after annealing, respectively. The inset is AFM image of sample. (c) PL spectrum of mechanical exfoliated monolayer $\mathrm{MoS}_{2}$ on $\mathrm{SiO}_{2}$ before annealing (orange curve) and after annealing in air (blue curve). Monolayer $\mathrm{MoS}_{2}$ is marked by white dash line in the inset

单层 $\mathrm{MoS}_{2}$ 样品 [图 2(c)]. 两个样品均在空气中 $250{ }^{\circ} \mathrm{C}$ 退 火 10 min. 经过空气退火后, 两个样品 $\mathrm{A}$ 激子荧光峰的 强度均产生了明显的增强, 并且峰位蓝移. 这也间接说 明了祄底和生长因素并不是空气退火导致荧光增强的
主要原因.

事实上，作为一种二维材料，单层 $\mathrm{MoS}_{2}$ 的电子分 布极易受到外界条件的影响, 导致荧光光谱性质的改 变. 有文献报道, 某些表面杂质可以改变二硫化钼的苂 光特性 ${ }^{[18,19]}$. 理论计算表明, 一些气体分子吸附在二硫 化钿表面能够引起电荷的转移 ${ }^{[20]}$. 例如, 当 $\mathrm{O}_{2}$ 分子吸 附在二硫化钼表面时, 能将电荷从二硫化钿的表面转移 走, 充当受主的角色; 而 $\mathrm{NH}_{3}$ 则充当电荷提供者的角色. 每个 $\mathrm{O}_{2}$ 分别可以转移走的电荷是 $0.041 \mathrm{e}$, 而每个 $\mathrm{NH}_{3}$ 能提供的电荷是 $0.069 \mathrm{e}$. 一般情况下, 未经处理的单层 二硫化钼是 $\mathrm{N}$ 型半导体，一部分电子被激子束缚后形成 负电激子. 负电激子的能量比中性激子能量低, 激发一 个中性 $\mathrm{A}$ 激子的能量为 $1.88 \mathrm{eV}$, 而激发一个负电激子 的能量为 $1.84 \mathrm{eV}^{[11]}$.

这里, 我们猜测, 空气退火导致了二硫化锄中缺陷 的形成, 这些缺陷使得大量的氧气被束缚(区别于室温 下氧气分子的吸附). 我们测量了空气退火前后器件的 输运性质(图 3). 退火后, 器件的电流下降了一个量级, 器件迁移率由 $11.5 \mathrm{~cm}^{2} \cdot \mathrm{V}^{-1} \cdot \mathrm{s}^{-1}$ 下降到 $0.94 \mathrm{~cm}^{2} \cdot \mathrm{V}^{-1} \cdot \mathrm{s}^{-1}$. 这证明了退火后大量缺陷的形成. 氧气分子的受主作 用, 改变了二硫化钿负电激子和中性激子的分布: 中性 激子增加、负电激子减少. 由于电荷的屏蔽作用, 负电 激子往往更难复合, 其荧光量子效率比中性激子低 ${ }^{[1]]}$. 因此, 我们在实验中看到空气中退火后峰位的蓝移和增 强. 我们对机械剥离的样品退火前后的荧光光谱分别进 行多峰拟合, 如图 4(a)所示, 荧光峰由 $\mathrm{A}, \mathrm{A}^{-}, \mathrm{B}$ 三激子 峰组成, $\mathrm{A}$ 激子与 $\mathrm{A}^{-}$激子能量差为 $40 \mathrm{meV}$, 在空气中退 火后 $\mathrm{A}$ 激子明显增强, 而 $\mathrm{A}^{-}$激子则明显减弱, 使得峰 位整体向中性激子峰偏移, 其峰移为 $20 \mathrm{meV}$ 左右. 这 也解释了图 1(d)和图(2b), (2c)中空气退火后发生的峰位 蓝移现象.

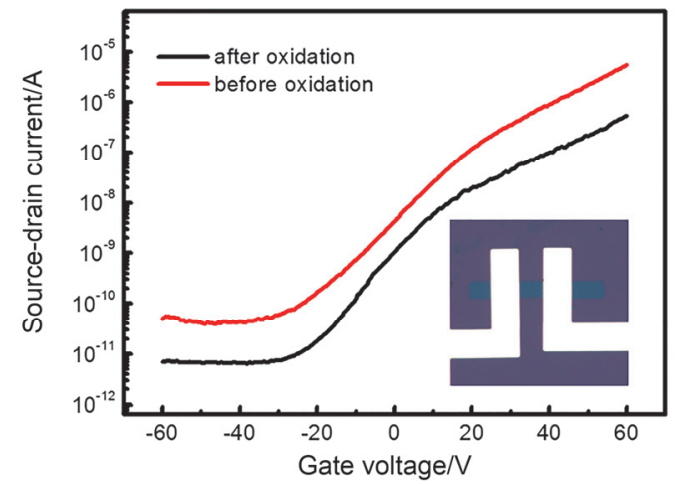

图 3 退火前后单层二硫化钿器件的转移曲线, 红色为退火前, 黑色 代表退火后. 右下角为器件的光学显微图片, 蓝色部分为 CVD 方法 生长在二氧化硅祄底上的单层二硫化钼. 器件在空气中 $150{ }^{\circ} \mathrm{C}$ 退火 5 $\min$, 可以明显看到退火后的电流大概下降了一个量级

Figure 3 Electrical transfer curve of device made by CVD grown monolayer $\mathrm{MoS}_{2}$ on $\mathrm{SiO}_{2}$. The inset is optical image of the device. Electrical measurement is carried out after the device is annealed in air at $150{ }^{\circ} \mathrm{C}$ for $5 \mathrm{~min}$. Electric current declines with an order of magnitude after annealing 

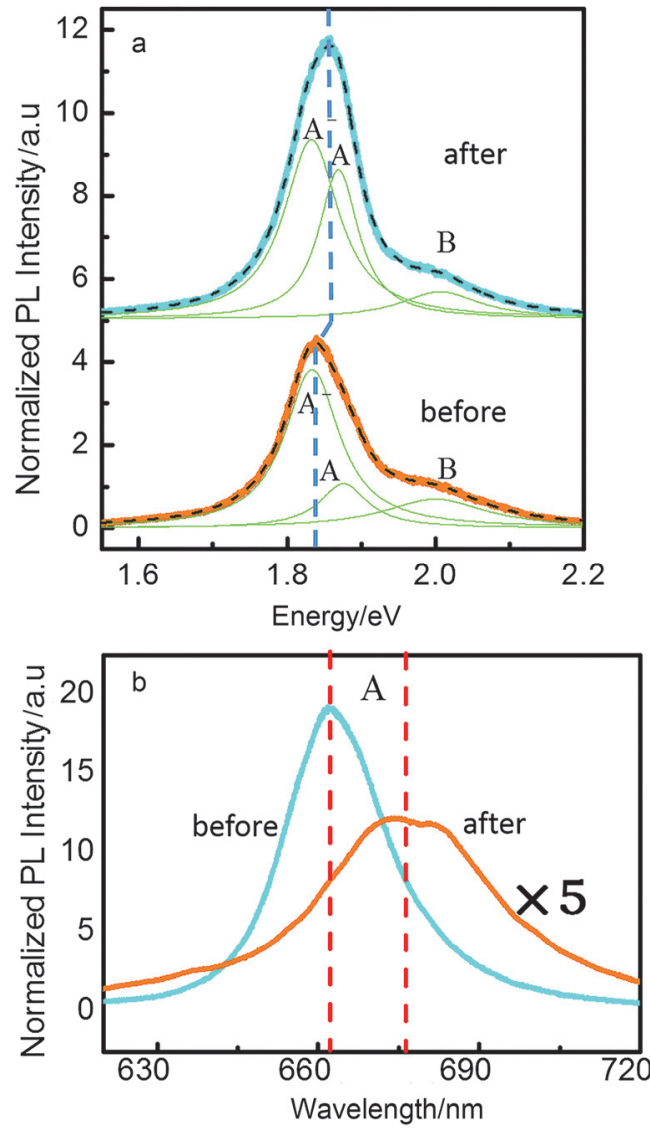

图 4 (a)机械剥离的单层二硫化钼样品在空气中退火前(橙色)和退火 后(蓝色)的荧光光谱的洛伦兹拟合谱, 整个光谱拟合为三个激子峰; (b) $\mathrm{CVD}$ 生长在 $\mathrm{SiO}_{2}$ 上的单层二硫化钼样品在 $\mathrm{NH}_{3}$ 吸附前(蓝色)和吸 附后(橙色)的苂光光谱, 其中橙色光谱被放大了 5 倍

Figure 4 (a) PL spectrum of $\mathrm{B}, \mathrm{A}$ and $\mathrm{A}^{-}$features is fitted to Lorentzians (green solid lines). (b) PL spectrum of CVD grown monolayer $\mathrm{MoS}_{2}$ with $\mathrm{NH}_{3}$ adsorbed (orange curve, which is magnified by 5 times) and non- $\mathrm{NH}_{3}$ adsorbed (blue). $\mathrm{MoS}_{2}$ is grown on $\mathrm{SiO}_{2}$ substrate

为了更好地验证我们的猜想, 我们进行了 $\mathrm{NH}_{3}$ 分子 吸附的对比实验 [图 4(b)]. $\mathrm{NH}_{3}$ 分子在二硫化钼表面为施 主型杂质，依据上述的猜想，当 $\mathrm{NH}_{3}$ 分子被充分吸附后， 二硫化钿中负电激子浓度得到增强, 峰位应当红移, 且 整体荧光强度下降. 我们将 CVD 生长的单层二硫化锄 样品置于压强为 300 torr 的 $\mathrm{NH}_{3}$ 中 $10 \mathrm{~h}$, 测量其荧光光 谱. 实验结果和预期相符: 荧光峰位红移, 强度下降.

考虑到过渡金属硫化物的苂光量子产率比较低 ${ }^{[5]}$, 空气(氧气)退火的方法为提高其荧光量子产率提供了一 种新的途径.

\section{3 结论}

我们对 CVD 方法生长在 $300 \mathrm{~nm} \mathrm{SiO}_{2}$ 上的单层二硫 化钿样品通过短时间的空气退火后, 观察到了 $\mathrm{A}$ 激子苂 光峰强度增强了大约一个量级, 荧光峰半宽减小, 峰位 整体有向高能区收缩的趋势. 这种空气退火对苂光的显 著增强的现象同样发生在机械剥离和 CVD 方法生长在
蓝宝石祄底上的单层二硫化钼样品上. 我们排除了祄底 和应力导致晶界开裂等因素的影响. 和氩气退火比较, 空气退火能更高效率地提高量子产率, 因此空气退火并 不是单纯地具有清洁表面和减少晶格缺陷的作用. 根据 他人相关的理论计算, 我们猜测, 空气中的氧气分子在 退火后被大量地束缚在二硫化钼表面, 起着受主掺杂的 作用. 当大量电子转移给氧气分子后, 二硫化钿中的负 电激子和中性激子的分布发生了明显的变化, 中性激子 大量增加, 负电激子急剧减少. 中性激子能量高、量子 产率高, 而负电激子能量低、量子产率低. 因此退火后 荧光强度明显增强, 峰位蓝移. 通过对退火前后荧光光 谱的洛伦兹拟合, 可以清楚地观察到这两种激子的变 化. 我们进行了 $\mathrm{NH}_{3}$ 分子吸附的对比实验. $\mathrm{NH}_{3}$ 分子为 施主型杂质, 能提高负电激子的数量, 导致退火后苂光 强度下降、峰位红移. 我们的实验结果和理论预计相符. 我们的研究提供了一种有效提高二硫化钿量子产率的 方案, 并具有气体探测和光电方面应用的潜在价值.

\section{4 实验部分}

样品的生长: 样品包括机械剥离和 CVD 生长的单 层二硫化钼. CVD 生长采用的是三温区真空管式炉. 其 中, $\mathrm{MoO}_{3}$ 粉末(Alfa Aesar 99.999\%)和硫粉(Alfa Aesar $99.9 \%$ ) 作为前驱物, 实验中的衬底分别为 $300 \mathrm{~nm}$ 厚 $\mathrm{SiO}_{2}$ 层的硅片和蓝宝石祄底. 两种前驱物和祄底分别置 于不同的温区，祄底位于管式炉气流的下流，载气(氞 气 $130 \mathrm{sccm}$ )携带前驱物在祄底上沉积. 通入载气前, 对 系统抽真空至 0.25 torr.

拉曼和荧光光谱: 实验的拉曼和荧光光谱在 Horiba Jobin Yvon LabRAM 高精度拉曼光谱仪上测量获得. 其激励光波长为 $532 \mathrm{~nm}$, 功率 $10 \mathrm{~mW}$, 光斑尺寸为 1 $\mu \mathrm{m}$.

表面形貌测试: $\mathrm{MoS}_{2}$ 样品的表面形貌在原子力显 微镜(MultiMode IIId, Veeco Instruments Inc)上完成, 实 验中使用 Tapping(轻敲)模式.

退火: 所有的退火均在单温区管式炉中完成.

器件的输运性质测量: 浓度为 5\%的 495PMMA 旋 涂在样品表面, 旋转速度 $4000 \mathrm{r} / \mathrm{min}$, 经 Raith e-beam lithography 系统加工形成带状. $\mathrm{MoS}_{2}$ 的刻蚀在反应离子 刻蚀系统中完成, 氩气等离子体作为反应刻蚀气体, 其 工作条件为功率 $50 \mathrm{~W}$, 氩气流量 $50 \mathrm{sccm}$, 气压 0.1 torr, 刻蚀时间 $20 \mathrm{~s}$. 金属电极的制备由电子束曝光和金属薄 膜沉积工艺完成. 器件的电学输运测量由安捷伦半导体 参数分析仪 $(4156 \mathrm{C})$ 完成, 测量平台为高真空四探针台 系统.

\section{References}

[1] Wang, Q. H.; Kalantar-Zadeh, K.; Kis, A.; Coleman, J. N.; Strano, M. S. Nat. Nanotechnol. 2012, 7, 699.

[2] Zhang, Y.-Q.; Liang, Y.-M.; Zhou, J.-X. Acta Chim. Sinica 2014, 72, 
367. (张芸秋, 梁勇明, 周建新, 化学学报, 2014, 72, 367.)

[3] Geim, A. K.; Novoselov, K. S. Nat. Mater. 2007, 6, 183.

[4] Chhowalla, M.; Shin, H. S.; Eda, G.; Li, L. J.; Loh, K. P.; Zhang, H. Nat. Chem. 2013, 5, 263.

[5] Splendiani, A.; Sun, L.; Zhang, Y.; Li, T.; Kim, J.; Chim, C.-Y.; Galli, G.; Wang, F. Nano Lett. 2010, 10, 1271.

[6] Mak, K. F.; He, K. L.; Shan, J.; Heinz, T. F. Phys. Rev. Lett. 2010, 105,136805 .

[7] Julien, C.; Sekine, T.; Balkanski, M. Solid State Ionics 1991, 48, 225.

[8] Late, D. J.; Liu, B.; Matte, H. S. S. R.; Dravid, V. P.; Rao, C. N. R. ACS Nano 2012, 6, 5635 .

[9] Li, H.; Yin, Z.; He, Q.; Li, H.; Huang, X.; Lu, G.; Fam, D. W. H.; Tok, A. Y.; Zhang, Q.; Zhang, H. Small 2012, 8, 63.

[10] Mak, K. F.; He, K.; Lee, G. H.; Hone, J.; Heinz, T. F.; Shan, J. Nat. Mater. 2013, 12, 207

[11] Tongay, S.; Zhou, J.; Ataca, C.; Liu, J.; Kang, J. S.; You, L.; Li, J.; Grossman, J. C.; Wu, J. Nano Lett. 2013, 13, 2831.

[12] Ross, J. S.; Wu, S.; Yu, H.; Ghimire, N. J. Jones, A. M.; Aivazian,
G.; Yan, J.; Mandrus, D. G.; Xiao, D.; Yao, W.; Xu, X. Nat. Commun. 2013, 4, 1474.

[13] Dolui, K.; Rungger, I.; Pemmaraju, C. D.; Sanvito, S. Phys. Rev. B 2013, 88,075420 .

[14] Peimyoo, N.; Yang, W.; Shang, J.; Shen, X.; Wang, Y.; Yu, T. ACS Nano 2014, 8, 11320.

[15] Lin, J. D.; Han, C.; Wang, F.; Wang, R.; Xiang, D.; Qin, S.; Zhang, X.-A.; Wang, L.; Zhang, H.; Wee, A. T. S.; Chen, W. ACS Nano 2014, 8, 5323.

[16] Zhang, J.; Yu, H.; Chen, W.; Tian, X.; Liu, D.; Cheng, M.; Xie, G.; Yang, W.; Yang, R.; Bai, X.; Shi, D.; Zhang, G. ACS Nano 2014, 8 , 6024

[17] Ji, Q.; Zhang, Y.; Gao, T.; Zhang, Y.; Ma, D.; Liu, M.; Chen, Y.; Qiao, X.; Tan, P.-H.; Kan, M.; Feng, J.; Sun, Q.; Liu, Z. Nano Lett. 2013, 13, 3870.

[18] Mouri, S.; Miyauchi, Y.; Matsuda, K. Nano Lett. 2013, 13, 5944.

[19] Eda, G.; Yamaguchi, H.; Voiry, D.; Fujita, T.; Chen, M.; Chhowalla, M. Nano Lett. 2011, 11,5111 .

[20] Yue, Q.; Shao, Z.; Chang, S.; Li, J. Nano Res. Lett. 2013, 8, 425.

(Lu, Y.) 